\title{
Expression Analysis of MiR-124 in Experimental Cerebral Hypoperfusion
}

\author{
Tang Li', Huizi Lei' ${ }^{2}$ Linhui Wang², Yu Li ${ }^{2 *}$ \\ ${ }^{1}$ Department of Pathophysiology, Chongqing Medical University, Chongqing, China \\ ${ }^{2}$ Department of Pathology, Institute of Neuroscience, Chongqing Medical University, Chongqing, China \\ Email: tanglicq@126.com, *liyu100@163.com
}

How to cite this paper: Li, T., Lei, H.Z., Wang, L.H. and Li, Y. (2018) Expression Analysis of MiR-124 in Experimental Cerebral Hypoperfusion. Journal of Biosciences and Medicines, 6, 9-14. https://doi.org/10.4236/jbm.2018.65002

Received: March 25, 2018

Accepted: May 18, 2018

Published: May 21, 2018

\begin{abstract}
MicroRNAs (miRNAs) are small noncoding single-stranded RNA molecules that act as negative regulators of gene expression and modulate the stability and/or the translational efficiency of target messenger RNAs. Studies have shown that miRNAs control diverse aspects of brain disease. In this study, the expression of miR-124 was investigated to explore the possible impacts of them on cerebral hypoperfusion. The model of aging rats with cerebral hypoperfusion was established by permanent occlusion of bilateral common carotid arteries (2VO). The expression of miR-124 was determined by real-time PCR. Cell cycle analysis was performed by fluorescence-activated cell sorting (FACS). Results showed that compared with control group, the expression of miR-124 decreased at early stage after operation in 2VO rats, the lowest level appeared on day $7(\mathrm{p}<0.05)$. Then the expression of miR-124 increased slightly on day 14. Overexpression of miR-124 in SH-SY5Y cell exposed to oxygen and glucose deprivation (OGD) induced cell cycle arrested in G1 phase. MiR-124 is potentially involved in cerebral hypoperfusion progression which may provide a novel therapeutic strategy for treatment of cerebral hypoperfusion.
\end{abstract}

\section{Keywords}

MiRNA-124, Cerebral Hypoperfusion, 2VO, OGD

\section{Introduction}

MicroRNAs (miRNAs) are 20- to 22-nucleotide RNAs that regulate the expression of multiple target genes by repressing translation and inducing mRNA degradations through binding to the 3 ' untranslated region (UTR) of target genes [1]. Negative regulation of gene expression by miRNAs occurs in a variety of bi- 
ological processes including differentiation, morphogenesis, proliferation and apoptosis. However, recent researches have also found that this regulation may be a positive activation [2]. MiR-124 (also called miR-124a) is the most abundant miRNAs in the brain. It is considered neuron specific because it is not expressed by astrocytes. Studies have shown that miRNA plays an important role in the development of ischemic brain injury [3]. In this study, the change of miR-124 expression and its role in cell cycle were observed in experimental cerebral hypoperfusion. The correlations were investigated to clarify the potential target for cerebral hypoperfusion treatment.

\section{Materials and Methods}

\subsection{Animals}

Fifty 12 -month-old ageing male Sprague-Dawley rats (450 to $500 \mathrm{~g}$ ) were obtained from the laboratory animal center of Chongqing Medical University (Animal license: No. SCXK 07-0001). Their use was approved by the Animal Ethics Committee of Chongqing Medical University.

\subsection{Experimental Design}

The model of ageing rats with cerebral hypoperfusion was established by permanent occlusion of bilateral common carotid arteries (2VO). In brief, after induction of anesthesia with chloral hydrate $(350 \mathrm{mg} / \mathrm{kg}$, intraperitoneal (i.p.)), both common carotid arteries were exposed through a midline ventral incision and permanently ligated with silk suture. The surgical wound was then sutured. After operation, alive rats were randomly divided into $2 \mathrm{VO}$ group $(1.7,14,21$ and 28 days after operation). The sham-operation group underwent an identical procedure without ligation of the bilateral common carotid arteries.

\subsection{Determination of Cerebral Cortex Blood Flow}

Rats were sacrificed and the brain was taken out completely. Image Launcher Software 1.4 Software was used to analyze brain tissue images of rats, and the changes of cerebral cortex blood flow were analyzed.

\subsection{Hematoxylin-Eosin (HE) Staining}

The brain was fixed with $4 \%$ PFA, dehydrated and embedded in paraffin. Two-micrometer frozen sections were prepared, and HE staining was performed to assess pathological changes in the CA1 regions of hippocampal tissue by light microscopy.

\subsection{Quantification of miR-124 Expression by Real-Time PCR}

The standard SYBR Green RT-PCR detection method was used for real-time PCR. Through CFX-96, real-time quantitative RT-PCR amplification was carried out. The threshold cycle $(\mathrm{Ct})$ values were automatically determined for each reaction by the Mastercycler EP Realplex real-time PCR system (Eppendorf) set 
with default parameters. U6 RNA was used as the internal reference.

\subsection{Cell Cycle Analysis}

SH-SY5Y cells transfected with pre-miR-124 by lipidosome to overexpress miR-124. SH-SY5Y-miR-124 cells and control cells were seeded in 6 well plate and cultured $24 \mathrm{~h}$. After that, cells were washed with PBS twice. Oxygen and glucose deprivation (OGD) was induced by incubating the cells for $30 \mathrm{~min}$ in a glucose and serum-free DMEM medium equilibrated with a $94.5 \% \mathrm{~N}_{2} / 0.5 \%$ $\mathrm{O}_{2} / 5 \% \mathrm{CO}_{2}$ atmosphere at $37^{\circ} \mathrm{C}$. Control group was cultured with DMEM containing glucose and 10\% FBS. Cells were harvested, fixed with ice-cold $70 \%(\mathrm{v} / \mathrm{v})$ ethanol and stored at $4^{\circ} \mathrm{C}$ overnight. Fixed cells were washed and resuspended in PBS and incubated with $20 \mu \mathrm{g} / \mathrm{mL}$ RNase A and $12 \mu \mathrm{l} 10 \times \mathrm{PI}$ at $4^{\circ} \mathrm{C}$ for $30 \mathrm{~min}$. PI staining was measured at $488 \mathrm{~nm}$ with a FACScalibur flow cytometer.

\subsection{Statistical Analysis}

All data analyses were conducted using SPSS17.0. Statistical comparisons were performed using the Student's t-test. The differences between groups were evaluated using one-way ANOVA, with $\mathrm{P}<0.05$ being considered significant.

\section{Results}

\subsection{Cerebral Blood Flow Altered in 2V0 Rats}

Compared with the sham-operation group, the blood flow in the cerebral cortex of $2 \mathrm{VO}$ rats altered significantly. The blood supply in the temporal region of the cerebral cortex decreased on day 7 and 14 after operation, and reached to the lowest level $(42 \%)$ on day $14(\mathrm{P}<0.05)$. Regenerated branch vessels formed in brain cortex on day 21 and day 28 , the blood supply increased but did not recover. The blood supply reached to control group level (96\%) on day 28 after operation.

\subsection{Expression of miR-124 in Ischemic Hippocampal Area Decreased in 2VO Rats}

Expression of miR-124 in $2 \mathrm{VO}$ group was less than that in sham-operation group. On day 1 after operation, the expression of miR-124 in 2VO group was $(0.47 \pm 0.04)$ times of sham-operation group $(\mathrm{P}<0.05)$. On day 7 after operation, the expression of miR-124 in $2 \mathrm{VO}$ group continued to decline and was $(0.27 \pm 0.03)$ times of sham-operation group $(\mathrm{P}<0.05)$. On day 14 after operation, the expression of miR-124 in $2 \mathrm{VO}$ group increased slightly, but still less than that in the sham-operation group. These results indicated that the expression of miR-124 was inhibited in hypoxia environment caused by cerebral ischemia, and it decreased to the lowest level on day 7 after operation. The expression of miR-124 gradually recovered to the sham-operation group level after 7 days. 


\subsection{Morphological Changes of Brain Tissue in 2VO Rats}

Due to cerebral hypoperfusion, pyramid neuron degenerated and lost in CA1 area on day 7 after operation, and several red neurons could be seen on day 14 after operation. The ischemic injury of the pyramidal neurons improved gradually on day 21 and 28 after operation, but did not restored completely (Figure 1).

\subsection{Overexpression of miR-124 Arrested Cell Cycle of SH-SY5Y Cells}

The analogue of miR-124 was transfected in SH-SY5Y cells and OGD was induced. The results showed that overexpression of miR-124 arrested cell cycle in G1 phase. After exposed to OGD for $24 \mathrm{~h}$ and $48 \mathrm{~h}$, SH-SY5Y-miR-124 cells in G1 phase increased $14 \%$ and $16 \%$, respectively $(\mathrm{p}<0.05)$ (Table 1$)$.

\section{Discussion}

MicroRNAs (miRNAs) are small non-coding RNAs that negatively regulate gene expression at the post-transcriptional level [4]. They are involved in important biological processes including development, homeostasis, and ageing. It is now evident that miRNAs are able to regulate the expression of at least one third of the human genome and play a critical role in a variety of normal biological processes, including cell differentiation, apoptosis, development, and metabolism. MiR-124 is the most abundant miRNAs expressed in the brain, it is enriched in the CNS with more than 100 times higher than that in other organs and accounting for more than a quarter of all brain miRs [5]. It is considered neuron specific because it is not expressed by astrocytes. The levels of miR-124 increase over time in the developing brain [6]. MiR-124 has been shown to play a key role in neuronal differentiation and neurogenesis [7]. The present in vivo studies revealed continuously downregulation of miR-124 in the hippocampus from acute to chronic phase of hypoxia as well as under $A \beta$ insult. However, few scholars have studied the role of miR-124 in cerebral ischemia.

Permanent bilateral common carotid artery occlusion or two-vessel occlusion $(2 \mathrm{VO})$ model is a classic model of cerebral hypoperfusion, and closely

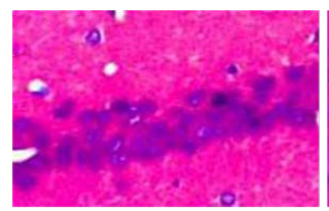

Control

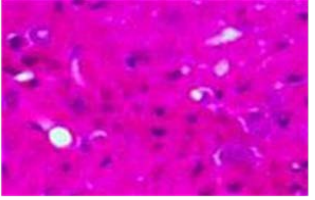

Day7

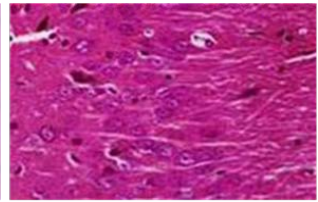

Day14

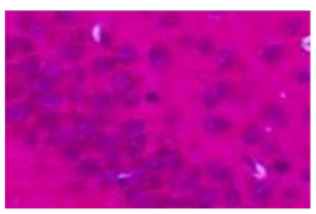

Day21

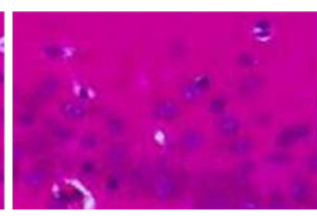

Day28

Figure 1. Morphological changes in hippocampal CA1 area (HE staining) $(\times 400)$. 
Table 1. Overexpression of miR-124 arrested cell cycle in SH-SY5Y cells.

\begin{tabular}{ccccc}
\hline & & \multicolumn{3}{c}{ Cell event (\%) } \\
\cline { 3 - 5 } & & G1 phase & S phase & G2-M phase \\
\hline \multirow{2}{*}{$24 \mathrm{~h}$} & Control & 69.7 & 14.6 & 15.7 \\
& Scramble & 67.2 & 16.4 & 16.2 \\
& Pre-miR-124 & 81.1 & 6.9 & 12 \\
& Control & 53.3 & 41 & 0 \\
& Scramble & 63.6 & 35 & 1.19 \\
& Pre-miR-124 & 75.8 & 19.3 & 4.85 \\
\hline
\end{tabular}

related with vascular dementia and Alzheimer's disease. In our study we found that there was a dynamic change of cerebral blood flow from decrease to increase in $2 \mathrm{VO}$ rats. The expression of miR-124 also showed this dynamic change, and the expression of miR-124 decreased to the lowest level on day 7 after operation $(\mathrm{p}<0.05)$. Due to the significant changes of miR-124 expression in the ischemic hippocampal area, we further explored the possible biological significance of it in sustained cerebral ischemia. It is evident that miR-124 played an important role in cell proliferation, oxygen-glucose deprivation is a commonly used model to mimic an ischemic milieu in vitro. So we established OGD model and transfected plasmid in SH-SY5Y cell to overexpress miR-124. The results showed that overexpress of miR-124 could arrest SH-SY5Y cell in G1 phase. It indicated that the decreased expression level of miR-124 might promote the cell cycle progression, thus played a neuroprotective role in the acute phase of ischemic brain injury.

In recent years, more and more studies have shown that the expression of miR-124 in ischemic brain injury was significantly altered, suggesting that miR-124 closely related to ischemic brain injury. Sun and his colleagues found the level of miR-124 was significantly increased in ischemic penumbra as compared with that in nonischemic area of middle cerebral artery occlusion (MCAO) mice. In vitro, miR-124 reduced neuron apoptosis and death induced by OGD, and antiapoptosis protein Bcl-2 and Bcl-xl increased, respectively [8]. Liu and his colleagues found that miR-124 downregulated inhibitory member of the apoptosis-stimulating proteins of $\mathrm{p} 53$ family (iASPP) and promoted neuronal death after cerebral ischemia [9]. Zhu and colleagues validated that knockdown of cerebral miR-124 could reduce cell death and infarct size and improve neurological outcomes [10]. Conversely, Doeppner and colleagues found that viral vector-mediated miR-124 delivery increased the resistance of cultured OGD cortical neurons in vitro and reduced brain injury [11]. Although these studies had an opposite result, and mechanism are unclear, miR-124 is still a potential target for treatment of cerebral ischemia or ischemia reperfusion injury and may provide a new method in clinic. 


\section{References}

[1] Ullah, S., John, P. and Bhatti, A. (2014) MicroRNAs with a Role in Gene Regulation and in Human Diseases. Mol Biol Rep, 41, 225-232.

https://doi.org/10.1007/s11033-013-2855-1

[2] Chan, S.H., Wu, C.W., Li, A.F., et al. (2008) miR-21 microRNA Expression in $\mathrm{Hu}$ man Gastric Carcinomas and Its Clinical Association. Anticancer Res, 28, 907-911.

[3] Jung, H.J. and Suh, Y. (2014) Circulating miRNAs in Ageing and Ageing-Related Diseases. J Genet Genomics, 41, 465-472. https://doi.org/10.1016/j.jgg.2014.07.003

[4] Bartel, D.P. (2004) MicroRNAs: Genomics, Biogenesis, Mechanism, and Function. Cell, 116, 281-297. https://doi.org/10.1016/S0092-8674(04)00045-5

[5] Krichevsky, A.M., King, K.S., Donahue, C.P., et al. (2003) A microRNA Array Reveals Extensive Regulation of microRNAs during Brain Development. RNA, 9, 1274-1281. https://doi.org/10.1261/rna.5980303

[6] Makeyev, E.V., Zhang, J., Carrasco, M.A., et al. (2007) The MicroRNA miR-124 Promotes Neuronal Differentiation by Triggering Brain-Specific Alternative pre-mRNA Splicing. Mol Cell, 27, 435-448. https://doi.org/10.1016/j.molcel.2007.07.015

[7] Yoo, A.S., Sun, A.X., Li, L., et al. (2011) MicroRNA-Mediated Conversion of Human Fibroblasts to Neurons. Nature, 476, 228-231. https://doi.org/10.1038/nature10323

[8] Sun, Y., Gui, H., Li, Q., et al. (2013) MicroRNA-124 Protects Neurons against Apoptosis in Cerebral Ischemic Stroke. CNS Neurosci Ther, 19, 813-819. https://doi.org/10.1111/cns.12142

[9] Liu, X., Li, F., Zhao, S., et al. (2013) MicroRNA-124-Mediated Regulation of Inhibitory Member of Apoptosis-Stimulating Protein of p53 Family in Experimental Stroke. Stroke, 44, 1973-1980. https://doi.org/10.1161/STROKEAHA.111.000613

[10] Zhu, F., Liu, J.L., Li, J.P., et al. (2013) Microrna-124 (mir-124) Regulates ku70 Expression and Is Correlated with Neuronal Death Induced by Ischemia/Reperfusion. $J$ Mol Neurosci, 52, 148-155. https://doi.org/10.1007/s12031-013-0155-9

[11] Doeppner, T.R., Doehring, M., Bretschneider, E., et al. (2013) MicroRNA-124 Protects against Focal Cerebral Ischemia via Mechanisms Involving Usp14-Dependent REST Degradation. Acta Neuropathol, 126, 251-265.

https://doi.org/10.1007/s00401-013-1142-5 